\section{4 \\ PHYSICAL AND CHEMICAL ASPECTS OF RUBBER TECHNOLOGY}

$\mathrm{T}$

HE Rubber Technology Conference, held in London during June 23-25 under the auspices of the Institution of the Rubber Industry, provided the first opportunity since the War for scientific workers and technologists in this field from many countries to meet for a general discussion of their problems. The papers presented covered a very wide range of subjects, from purely scientific to highly technical; this short article is an attempt to review only the more scientific aspects of the matters discussed.

\section{Physical Aspects}

It has been known for a long time that natural rubber crystallizes on stretching, and it is generally supposed that this phenomenon has an important bearing on such mechanical properties as tensile strength and resistance to tearing. In a paper on the degree of crystallization in natural rubber, Dr. J. M. Goppel discussed his recent X-ray studies of the absolute amount of crystallization as a function of the degree of extension of the rubber. For a highly extended vulcanized rubber, the fraction of crystalline material was found to be only 29 per cent, which is very much lower than figures given by the earlier work of Field (60-80 per cent). Correspondingly low figures were obtained for frozen raw rubber (unstretched), for which the degree of crystallization was found to increase with time of storage. The highest figure obtained was 38 per cent for crepe rubber which had been stored for twenty-nine years. It was shown also that estimates of crystallinity from density changes reported in the literature led to values of the same general magnitude.

Papers dealing with the design of engineering components in rubber were contributed by Mr. R. S. Rivlin, who dealt with the theoretical basis of this subject, and by Mr. H. L. Jenkins and Mr. H. D. Cooper, whose approach was from the experimental side. Mr. Rivlin referred to the inherent limitations of the classical theory of elasticity-particularly the restriction to small deformations-and gave an outline of recent developments in the theory of large elastic deformations, with particular reference to incompress. ible materials, that is, materials like rubber, the volume of which is substantially constant on deformation. Rivlin's theory is centred on the existence of a unique work of deformation or stored-energy function corresponding to a specified state of strain; from this stored-energy function the generalized stress-strain relations are readily derived. The theory has been applied to a number of problems of practical interest, among which shear mountings and torsional couplings were particularly considered.

From experiments on the axial compression of cylindrical blocks between lubricated plates (to allow free lateral expansion), Mr. Jenkins and Mr. Cooper showed that the properties of rubbər in compression can be adequately represented by the formula derived from the statistical theory of James and Guth. This formula, which had previously been applied mainly to rubbər in tension, involves only one physical constant $k$, which is analogous to the modulus of rigidity in the classical theory. The experimental values of $k$ are nearly independent of the ratio of length to diameter of the cylinder. In the case of shear, the theoretical
August 7, 1948 Vol. 162

linear relation between stress and strain was found to be inadequate; but the experimental results can be represented by the use of the same constant $k$, together with an empirical 'shape function'. It was pointed out, however, that the values of $k$ are dependent on the time of loading or 'load history' adopted, and a machine was described which has been designed to facilitate the application of different load-history patterns.

Another aspect of the mechanical properties of rubber, the rate of retraction from the stretched state after release of the tension, was examined by Dr. B. B. S. T. Boonstra. The technique employed depends on the breaking of electrical contacts by the motion of the rubber, the time between successive breaks being measured by the fall in potential of a condenser discharging through a resistance. Differences were found both in the rates of retraction, and in the residual temporary 'set', between the various rubbers (natural and synthetic) examined. The speed of retraction did not appear to be correlated with rebound resilience; for example, butyl rubber, though showing very low rebound resilience, gave a retraction-rate only slightly below that for natural rubber.

The two sessions devoted to the discussion of compounding ingredients included a number of papers dealing with the effects of fillers, particularly carbon black, on the physical properties of rubber. Although carbon black is regarded as the outstanding 'reinforcing' filler for rubber, there appeared from the discussion to be no general agreement as to what constitutes reinforcement, though tensile strength, stiffness and abrasion resistance were the most frequently quoted criteria. In view of this lack of definition of the concept, it is not surprising to find that the mechanism of reinforcement is still but imperfectly understood. A new and interesting fundamental approach to this problem was provided by Dr. W. R. Smith and Mr. W. D. Schaeffer, who have attempted to evaluate the surface activity of various carbon blacks by measurements of the heats of adsorption of gaseous hydrocarbons. Their results reveal a marked variation in heats of adsorption with degree of surface covering (up to 1.5 molecular layers), which points to a selective adsorption at specially active sites. How far these conclusions are directly applicable to the carbon-rubber system has yet to be established; but the evidence suggests that there is a rather close correlation between the initial heats of adsorption and the reinforcing power of the black.

A general physical approach was adopted in the paper by Dr. E. Guth on the theory of filler reinforcement. Guth's theory concentrates attention on the effect of included rigid particles on the modulus of an elastic medium. This theory leads to an expression for the modulus in terms of the volume concentration of the filler which may be regarded formally as an extension of the well-known Einstein relation for the viscosity of a liquid medium containing solid particles in suspension. By means of this equation it should be possible to distinguish between approximately spherical particles, and particles possessing marked geometrical anisotropy. The experimental evidence quoted by Dr. Guth indicated that non-reinforcing fillers like calcium carbonate correspond to the case of spherical particles, while reinforcing fillers require the assumption of highly elongated particles. Since individual carbon particles are roughly spherical in shape, this is regarded as evidence for the formation of elongated chain-like agglomerates of particles, such 
as have frequently been observed in the examination of blacks under the electron microscope. Indeed, by a suitable modification of the theory it is possible to include the effect of non-sphericity in terms of a 'shape factor'; the shape factors thus derived are found to agree reasonably well with the shapes observed in the electron microscope. This conclusion received further support from Dr. L. H. Cohan, who presented electron microscope evidence for the existence of chain-like structures, which appear to be of characteristic form for any particular mode of preparation of the black. A large number of measurements on a particular black yielded an axial ratio of 6 , and this value was found to account for the observed increase of modulus, on Guth's theory, up to 25 per cent concentration.

This theory of reinforcement, as the subsequent discussion showed, is not without its difficulties. According to Dr. L. Mullins, the stiffening effect of carbon black 'wears off' after a small number of repetitions of the extension of the rubber, and gradually reappears on resting, suggesting a breakdown of the chain structures due to stressing. This aspect was further discussed by Mr. A. F. Blanchard and Dr. D. Parkinson, who made use of measurements of electrical conductivity, which is directly related to a net structure of the carbon particles, to throw light on the breakdown and re-formation of these structures. Their conclusion is that the degree of breakdown of structure on extension is relatively of a minor character, certainly not sufficient to account for the observed reduction of stiffness, which they attribute rather to the rupture of the bonds between the carbon particles and the rubber.

A contribution to the study of the optical properties of rubber was presented by Dr. L. A. Wood and Mr. L. W. Tilton, who described an accurate method of measuring refractive indices, in which the specimen was moulded into prismatic form between glass plates. Data were obtained at five different wave-lengths, and over a range of temperature, and compared with various theoretical formulæ for optical dispersion. An interesting result which emerged is the disparity between the isoprene unit and normal mono-olefins, which suggests that the middle $\mathbf{C}-\mathbf{C}$ bond may partake of the properties of a double bond. A corresponding abnormality in bond-length was found from an X-ray analysis by Jeffrey of the closely comparable low-molecular crystalline compound, geranylamine hydrochloride.

The curious luminescence which may sometimes be observed on tearing rubber in a dark room was the subject of a paper contributed by Dr. A. van Rossem. From numerous experiments it has been shown that the emission of light is related to the type of filler with which the rubber is compounded. The effect was observed only in the presence of nonreinforcing fillers. The suggestion was made that the phenomenon is due to a discharge of electricity through the vacuoles which are known to be formed in such filled rubbers under tension.

On an entirely different subject, Prof. H. W. Melville and Mr. C. R. Masson reviewed recent techniques for the measurement of very small osmotic pressures, as encountered in the determination of molecular weights of high polymers. The difficulty of such measurements arises from the length of time required for the attainment of osmotic equilibrium. It was shown that this may be greatly reduced $(a)$ by the use of bacterial cellulose as a semi-permeable membrane, and $(b)$ by the use of a dynamic method involving the measurement of the rate of approach to equilibrium. Another technique described was the 'osmotic balance' method, in which the diffusion of liquid into the cell is determined by weighing instead of by measurement. This method was claimed to give remarkable sensitivity, and should prove useful for the determination of molecular weights hitherto considered to be beyond the range of investigation by osmotic methods.

Among a number of papers dealing mainly with the technical evaluation of the properties of rubbers, many questions of interest to the physicist were touched on. Particular mention may be made of two papers on the resistance of rubber to tearing, by Dr. J.M. Buist and Dr. H. A. W. Nijveld, respectively, and one by Dr. J. R. Scott and Mr. R. W. Whorlow on the interpretation of plasticity measurements.

\section{Chemical Aspects}

Although most aspects of the chemistry of elastomers were implicit in the many matters discussed from a technological point of view, the actual contri. butions to rubber chemistry were limited to the three main topics of oxidation, vulcanization and rubber halides. There were also some subsidiary advances in chemical knowledge in the more technical discus. sions on synthetic rubbers and on latex.

On the subject of oxidation, papers presented by Dr. R. L. Stafford and by Dr. J. Le Bras and Mr. A. Salvetti were both primarily concerned with the mode of action of inhibitory substances. It has been suggested that in rubber chemistry the latter fall into two distinct classes, termed antioxidants and deactivators respectively, typical examples being phenyl- $\beta$-naphthylamine and mercapto-benzimidazole. The former fulfil the normal function of retarding oxygen attack, whereas the latter reduce the deleterious chain-scission reactions associated with oxidation of rubber without lessening the overall combination of oxygen. Much of the earlier work on the autoxidation of rubber and allied substances has been carried out with strong sources of light (for example, ultra-violet), thus giving a reaction which proceeds at a rate many times that of the dark reaction and which is unretarded by the usual antioxidants. No fundamental change in the nature of the reaction or of the effect of antioxidants was encountered by Dr. Stafford when the oxidation was effected with a weak light source with the object of obtaining a rate of photo-activated absorption of the same order as that of the dark thermal reaction.

However, although neither phenyl- $\beta$-naphthylamine nor mercapto-benzimidazole (the antioxidant and deactivator studied) was effective against lightactivated absorption, a mixture of the two was found to possess considerable efficacy. The precise mech. anism of this effect, which is in accord with earlier work of Le Bras, is still unsolved.

For the study of these oxidation effects with rubber, Dr. Le Bras has designed an improved type of manometric apparatus in which the oxygen is generated as required by electrolysis of a concentrated solution of copper sulphate.

The chief difficulty in this type of work is the fluctuation in results occasioned by the variability of composition of crude natural rubber. Assistance in this direction was given by Mr. G. Martin, who described a simplified method of preparing pure rubber hydrocarbon for oxidation studies. The method involves addition, to the ammonia-preserved latex, of a soap, which displaces the protein from the 
rubber particle surfaces, followed by repeated creaming, coagulation with acetic acid and extraction with acetone.

Considerable interest was shown in a paper by Dr. W. T. Chambers on the direct determination on a micro-scale of oxygen in rubbers. His method is an application of that of Unterzaucher involving reduction of the oxygenated compound with carbon, the resulting carbon monoxide being oxidized by iodine pentoxide and the liberated iodine estimated after oxidation to iodate. The ability to determine small percentages of oxygen directly, instead of by difference from the carbon and hydrogen figures, will be of great value in rubber oxidation studies. The accuracy of this method is of the order of \pm 0.01 in the range $0-0.5$ per cent oxygen and \pm 0.04 in the range 1.0 to $2 \cdot 0$ per cent oxygen. This precision was not sufficient to show conclusively that the molecular breakdown of rubber on milling is always associated with oxidation, but Dr. Chambers was able to demonstrate this effect in one run with pale crepe rubber.

In his presidential address to the Conference, Sir Robert Robinson mentioned the advances that had been made in the knowledge of polymerization and the importance of the introduction of intermolecular cross-linking into natural and synthetic polymers. Dr. G. F. Bloomfield was able to present more evidence on the nature of the cross-links arising in the vulcanization of rubber with sulphur. In this connexion methyl iodide has proved a useful reagent for determining the type of $\mathrm{C} \longrightarrow \mathrm{S}$ link present. Earlier work has shown that methyl iodide at $25^{\circ} \mathrm{C}$. removes the sulphur from diallylic sulphides to form trimethyl sulphonium iodide, and since the latter is obtained from the reaction of methyl iodide with rubber/sulphur vulcanizates, it has in the past been assumed that diallylic groups must be present in appreciable numbers. The unreliability of this conclusion (which is, in any event, at variance with other vulcanization evidence) was made apparent by Dr. Bloomfield's demonstration of a similar formation of trimethylsulphonium iodide from compounds containing the sulphur atom between one allylic and one tertiary grouping. It is known that the reaction of polyisoprenic compounds with sulphur leads to the formation of cyclic and cross-linked sulphides, and the evidence presented in this work is consistent with the view that the allyl-tertiary sulphide grouping is a characteristic of both.

The importance of the non-hydrocarbon constituents of natural rubber in the process of vulcanization was demonstrated by Mr. G. Martin in his work on highly purified rubber. The catalytic effect of these other constituents, the absence of which was found markedly to reduce the vulcanizing efficiency of sulphur, is a subject requiring further careful investigation.

A study of the hydrochlorination of rubber in latex by Dr. G. J. van Veersen had theoretical as well as practical significance. The reaction of hydrogen chloride with rubber in solution shows an induction period, the length of which is proportional to the solubility of the hydrogen chloride in the solvent. It was suggested that a solution of rubber in dioxane (for example) represents a system of two electron donors competing for the hydrogen chloride to form co-ordination complexes. During the induction period, the speed of the reaction would be dependent on the affinity of the solvent for hydrogen chloride, but after saturation the rate would be independent of the nature of the solvent. In the reaction of hydrogen chloride with rubber latex it is necessary to prevent coagulation by reversing the charge on the rubber globules with a cationic soap. Under these conditions a very clean addition takes place with a minimum of cyclization.

It is difficult in a substance of high molecular weight like rubber to determine the nature of artificially introduced chemical groups. A contribution to this subject was made by Dr. G. Salomon, who read a paper by himself and Drs. C. Koningsberger and A. J. Ultée on the kinetic analysis of rubber halides. The method is based on the marked differences in reactivities of the various classes of aliphatic halides with organic bases. In the case of monohalides, this order is allylic > primary $>$ tertiary (with some exceptions) $>$ secondary $>$ secondary monocyclic $>$ vinylic. Polyhalides are more difficult to distinguish unambiguously, but their identification is rendered possible in many cases by comparison of the speeds of reaction with various bases. Using this kinetic method, evidence was found, for example, for the formation of secondary allylic chloride groupings in the chlorination of rubber under conditions favouring substitution, and of a series of polychlorides, the constitution of which has yet to be settled, by conventional chlorination.

The papers and discussion on synthetic rubbers were mainly technological in nature, although there were some points of chemical interest. Dr. R. P. Dinsmore directed attention to the fact that $G R-S$ requires less combined sulphur than natural rubber to give a satisfactory vulcanizate, a point that may well be connected with the tendency of the $\Delta^{1: 5}$ polyene system of rubber to give cyclic sulphides which contribute little to the strengthening of the molecular network. Mention was made of some of the physical and chemical properties associated with the characteristic structures of silicone, 'Buna- $N$ ' and butyl rubbers and of condensation rubbers of the type of 'Vulcaprene' (a di-isocyanate-modified polyesteramide).

An important contribution to our knowledge of the constitution of natural rubber latex was made by Miss L. N. S. Homans and Dr. G. E. van Gils. By centrifuging fresh unammoniated latex they were able to separate a yellow fraction which was a suspension mainly of non-rubber particles and which contained 6-8 per cent of the total solids of latex. These globules (termed 'lutoids') were easily visible under the microscope as transparent masses considerably larger than the rubber globules and were shown to consist of 80-85 per cent of water, with the solid content made up of proteins, salts and acetonesoluble substances, in the form of lipoids. Addition of ammonia brought the lutoids into solution, and dilution with water caused shrinking; but they were quite stable towards $0 \cdot 1 \mathrm{~N}$ sodium chloride in aqueous solution. Removal of the lutoids resulted in marked changes in properties of the latex; for example, greater susceptibility to photoxidation, lower mechanical stability, greater thermal stability.

This new knowledge may have interesting biological implications in that it brings the constitution of latex more into line with the complexity of other physiolog. ical liquids.

A further attraction of the conference was an exhibition, arranged by the Dunlop Rubber Company, which included demonstrations of some of the more striking of the properties of rubber and methods by which they have been studied. 
It is intended that the Proceedings of the Conference shall be published in a single volume. There is no doubt that this will prove a worthy companion to the Proceedings of the first conference, held in 1938, and a valuable source of reference to all interested in rubber seience.

L. R. G. TreLOAR

R. F. NAYLOR

\section{NUTRITION OF ATHLETES}

A WHOLE-DAY meeting of the Nutrition Society on July 17 was devoted to the subject of the "Nutrition of Athletes". The first papers dealt with the physiology of muscular effort, and the meeting took a more practical turn later in the morning and in the afternoon session.

The meeting opened with a paper by Dr. Philip Eggleton of Edinburgh on "The Neuromuscular Mechanism". Physiological studies, he said, fall into three clear-cut categories. The first of these is the work on animals (including dog and man) which comprises measurements of work done, respiratory measurements, urine and blood analyses, nutrition studies and so on. The second category includes work on isolated muscles and nerves, which has been carried out mainly on cold-blooded animals because of the difficulty of using such preparations from mammals. Thirdly, there is work on the muscles of marine shell-fish. These last two methods have certain disadvantages in that it is difficult to apply the results obtained from them to the mammal, and particularly to the human being. Nevertheless, results from the three fields combine to produce a picture of the neuro-muscular machine which, according to the author, comes nearest to the physiologist's ideal-a description of physiological phenomena in terms of chemistry and physics - than has been achieved in respect of any branch of physiology, save perhaps the transport of gases by the blood.

Experiments in the first field have shown a simple relation between work done and food intake required ; and there is also considerable evidence that manual labourers do not choose to eat carbohydrates but prefer fat and protein. This preference for a high protein diet by both manual labourers and athletes may be a survival prrhaps of sympathetic magic ; yet it may have as a basis the fact that meat is a good source of some of the B complex of vitamins. The preference for fatty food is more understandable, since it is an economical source of energy.

Dr. Eggleton went on to discuss A. V. Hill's work on the oxygen consumption during exercise, and said that in Hill's experiments the consumption of oxygen was about 4 litres a minute for men engaged in running at a pace which did not give them undue respiratory distress. However, these experiments only lasted for minutes, and Eggleton pointed out that the heart cannot pump 4 litres of oxygen into the body indefinitely ; if the exercise has to be maintained for some time, an oxygen consumption of 1 litre per minute is nearer the upper limit. In this connexion Dr. Eggleton remarked that an athlete can build up an oxygen debt for events such as the 100-yd. sprint, and that in this event the rate of output of energy of the runner is 10-13 horse-power, which corresponds to an oxygen intake of 23 litres a minute-six times anything the heart can achieve. This debt is financed partly by the breakdown of glycogen to lactic acid and partly by the reserve of non-oxidative energy represented by the creatin phosphate content of muscle.

Dr. Eggleton discussed some of the aspects of the evolution of the neuro-muscular mechanism, and pointed out that one of the differences between invertebrate and vertebrate muscle is the presence of arginine in the muscles of the former and of creatine in muscles of the latter. Also vertebrate muscles contain considerable amounts of two di-peptides$\beta$-alanylhistidine and $\beta$-alanyl-methyl-histidinewhich are not present in invertebrate muscle.

In conclusion, Dr. Eggleton commented upon the apparent increasing athletic abilities of the human race, which he felt were more apparent than real, and upon the view that the decreased performances of British athletes recently is due to the changes in diet and habits engendered by the War, a problem which he felt should be left to later speakers.

Dr. Dorothy Needham spoke on chemical aspects of chemical contraction. In this field, Dr. Needham said that two lines of work, followed independently for about half a century, had only recently come together. One of these was the nature of the muscle machine, and the other was the nature of the essential fuel. Dr. Needham discussed the early work of Fletcher and Hopkins and of Meyerhof on the energy liberated by the formation of lactic acid in muscle, and of Lundsgaard's work on creatine-phosphate breakdown as a source of energy. Lohman's work in 1934, she said, suggested that adenosine triphosphate hydrolysis supplied the first energy-yielding reaction in muscular contraction.

The old idea that lactic acid production is an inevitable accompaniment of contraction has had to be discarded since it has been shown that much work can go on without any lactic acid formation. The most important constituent of the muscle machinery is the protein, and the modern phase began with the work of Edsall and von Muralt in 1930. Solutions of myosin showed the property of double refraction in flow, and this suggested some relation with the anisotropic bands of myo-fibrils. It appears that the contractile fibrils of muscle consist of polypeptide chains of myosin showing the $\alpha$-fold, and owe their elasticity and contractility to their power of straightening out to the fully extended chain or folding up still further. The link between the machinery and the fuel comes from the observation that myosin has adenosine triphosphatase activity, so that myosin is not only the chief structural entity of muscle but is also the enzyme catalysing the chemical processes which lead to its own contraction. Szent-Györgyi has shown that another fibrous protein, 'actin', can be extracted from muscle, and that actin and myosin may unite to form fibrils of much greater length; this compound of the two proteins is called actomyosin.

It seems possible that some of the symptoms of fatigue may be due to the breaking of inter-molecular links which hold the partly folded polypeptide chains in place. If contraction is too severe and prolonged, too great a distortion may take place and unusual breaking of links or the formation of new and inconvenient ones may take place.

There are at the moment two main views on muscular contraction. One is that the contracting fibrils are made up of actomyosin. If adenosine triphosphate comes into contact with actomyosin fibrils, there is a rapid change of the protein with loss of water. Szent-Györgyi regards this as synonymous with contraction; on the other hand, Astbury has sug. gested that in resting muscle the fibrils are made 